\title{
Fentanyl compared to buprenorphine for atrial fibrillation ablation analgesia and sedation: a retrospective cross-sectional study
}

Teruhisa Kinoshita ${ }^{{ }^{*}}$ DD, Mitsunori Harada², Norio Takimoto', Daichi Shibata', Takashi Sakakibara ${ }^{1}$ and Mamoru Adachi ${ }^{1}$

\begin{abstract}
Background: The effects of general anesthesia with deep sedation and conscious sedation have been compared for sedation management in the perioperative period for radiofrequency catheter ablation of the heart to treat atrial fibrillation. However, there is no consensus as to which drug to use for conscious sedation. This study aimed to investigate analgesic and sedative drugs suitable for perioperative ablation.

Methods: We retrospectively examined 93 patients who underwent atrial fibrillation ablation at Kariya Toyoda General Hospital between December 2017 and April 2019 and investigated differences in the outcomes, such as depth of sedation and postoperative adverse events between the buprenorphine hydrochloride $(n=46)$ and fentanyl citrate $(n=47)$ groups.
\end{abstract}

Results: The depth of sedation was similar between the two groups, without significant between-group differences in postoperative vomiting. The number of additional injections of thiamylal sodium to manage discomfort and pain during ablation were significantly lower in the fentanyl group. Additionally, the cumulative area product, cumulative total air kerma, 1-year postoperative atrial fibrillation recurrence rate, and postoperative complications were not significantly different between the two groups.

Conclusions: Although there were no significant differences in the efficacy or safety between buprenorphine hydrochloride and fentanyl citrate as analgesics used during atrial fibrillation ablation, intraoperative body movements and patient discomfort could be reduced to a greater extent with the use of fentanyl.

Keywords: Atrial fibrillation, Buprenorphine hydrochloride, Catheter ablation, Fentanyl citrate

\section{Background}

Ablation for atrial fibrillation (AF) is considered effective for maintaining the sinus rhythm and has been shown to improve quality of life without increasing the risk of complications compared with medical therapy in the

\footnotetext{
*Correspondence: teruhisa.kinoshita@toyota-kai.or.jp

'Department of Pharmacy, Kariya Toyota General Hospital, 5-15, Sumiyoshi-cho, Kariya City, Aichi Prefecture 448-8505, Japan

Full list of author information is available at the end of the article
}

wider AF population, with additional mortality and clinical benefits in patients with heart failure.

It has also been suggested that ablation may reduce the risk of ischemic stroke and death [1-3], and therefore, it is widely used. Stable sedation/analgesia for ablation increases patient satisfaction and the success rate of the procedure and is considered essential for preventing complications such as cardiac tamponade and air embolisms. It has been reported that, in AF ablation, there

(c) The Author(s). 2021 Open Access This article is licensed under a Creative Commons Attribution 4.0 International License, which permits use, sharing, adaptation, distribution and reproduction in any medium or format, as long as you give appropriate credit to the original author(s) and the source, provide a link to the Creative Commons licence, and indicate if changes were made. The images or other third party material in this article are included in the article's Creative Commons licence, unless indicated otherwise in a credit line to the material. If material is not included in the article's Creative Commons licence and your intended use is not permitted by statutory regulation or exceeds the permitted use, you will need to obtain permission directly from the copyright holder. To view a copy of this licence, visit http://creativecommons.org/licenses/by/4.0/ The Creative Commons Public Domain Dedication waiver (http://creativecommons.org/publicdomain/zero/1.0/) applies to the data made available in this article, unless otherwise stated in a credit line to the data. 
was increased success in pulmonary vein isolation with general anesthesia than with conscious sedation $[4,5]$. However, there are facilities where it is difficult for the anesthetist to be present at the time of the ablation; therefore, conscious sedation is often carried out. Furthermore, there is no established consensus regarding which drug to use for perioperative anesthesia management for ablation. Even in the guidelines [6], there are no recommendations for specific drugs.

At the Kariya Toyota General Hospital from December 2017 to July 2018, anesthesia management was carried out using the following drugs: thiamylal sodium, dexmedetomidine hydrochloride, and buprenorphine hydrochloride. However, buprenorphine hydrochloride was switched to fentanyl citrate in August 2018 because of the high frequency of additional use of thiamylal sodium to reduce body movement caused by pain and intraoperative discomfort during myocardial cautery and reduce the high incidence of postoperative vomiting.

In the present study, we compared the efficacy of buprenorphine hydrochloride and fentanyl citrate and investigated analgesic and sedative drugs suitable for perioperative ablation.

\section{Methods}

\section{Target patients}

The study period was 17 months from December 1, 2017, to April 30, 2019. Eligible patients were those who had undergone ablation for AF at our hospital. The group treated with thiamylal sodium, dexmedetomidine hydrochloride, and buprenorphine hydrochloride was the "buprenorphine group," and the group treated with thiamylal sodium, dexmedetomidine hydrochloride, and fentanyl citrate was the "fentanyl group."

\section{Investigation method}

From electronic patient charts, we retrospectively analyzed patient characteristics including age, sex, body mass index (BMI), CHADS2 score, CHA2DS2-VASc score, serum creatinine, creatinine clearance $(\mathrm{Ccr})$, left atrial dimension (LAD), left ventricular ejection fraction (LVEF), and cardiac ablation site. Ccr was calculated using the Cockcroft-Gault equation: Ccr $(\mathrm{mL} / \mathrm{min})=$ $(140$-age $) \times$ weight / $(72 \times$ serum creatinine value) (for female patients, multiplied by 0.85 ).

Indicators of the effectiveness of ablation included the rate of AF recurrence at 1 year after ablation, postoperative complications, intraoperative cumulative dose area product, and cumulative total air kerma.

Intraoperative sedation was measured using the bispectral index (BIS) [7] and Richmond Agitation-Sedation Scale (RASS), which are defined below.

The BIS is a processed electroencephalographic parameter, which provides a measure of sedation depth on a unitless scale from 0 to 100 (0-40, deep hypnotic state; 40-60, general anesthesia; 60-90, deep-to-light sedation; and 90-100, awake).

The RASS describes the clinical level of sedation $(-5$, coma; -4 , deep sedation; -3 , moderate sedation; -2 , mild sedation; -1 , somnolent state; 0 , clarity of consciousness; 1 , restless; 2 , excited; 3 , very excited; 4 , belligerent), and has been used particularly for assessing the safety of different analgesics.

Side effects were recorded, including the presence of vomiting and the administration of antiemetics. Metoclopramide hydrochloride $10 \mathrm{mg}$ was administered intravenously as a prophylactic at the discretion of the attending physician. Vomiting was included as a side effect if it occurred after the beginning of treatment on the day of ablation.

Patients were followed for 1 year postoperatively to detect recurrence of AF. Patients with post-ablation complications that required prolonged hospital stay or hospitalization within 1 month and required treatment were considered to have complications.

For other sedatives, dexmedetomidine hydrochloride was administered continuously, and thiamylal sodium was temporarily added for the management of body movements and discomfort associated with the procedure. Doses of both drugs were determined at the discretion of the operators.

Sedation for intraoperative electrical defibrillation was performed with thiamylal sodium. To ascertain the number of doses of thiamylal sodium in response to body movements and discomfort caused by the procedure, thiamylal administration at the time of esophageal temperature sensor insertion and before electrical defibrillation was excluded from the count.

Intraoperative sedation was monitored by the cardiologist, and no anesthesiologist was present in the angiography room.

\section{Statistical methods}

For statistical analysis, we used EZR version 3.4.18 (Jichi Medical University Saitama Medical Center, Japan) [8]. Continuous variables are all shown as mean \pm S.D. For a comparison of continuous variables, after confirming the normality and distribution of the data, the Mann-Whitney $U$ test and Student's t-test were used for two-group comparisons, as appropriate. For a comparison of nominal variables, Fisher's exact test was used. Statistical significance was set at $p<0.05$.

\section{Ethical considerations}

This study was carried out following the Helsinki Declaration, the "Medical Guidelines for Medical and Health Research Involving Human Subjects," and the "Guide for the appropriate handling of personal information for 
medical and nursing care professionals." Approval was obtained from this hospital's Ethics Review Committee, and adequate consideration was given for the protection of personal data (Approval No. 517).

\section{Results}

\section{Patient characteristics}

We analyzed the data of 46 patients in the buprenorphine group and of 47 patients in the fentanyl group who underwent $\mathrm{AF}$ ablation at our hospital during the study period. The characteristics of the target patients are shown in Table 1.

Although LVEF was significantly higher in the buprenorphine group, it was with normal parameters in both groups, and there were no significant differences in terms of other parameters. The patients were relatively young (the average patient age was 64 years old), and their renal function was normal. Most patients had a relatively low risk of stroke caused by AF.

There was no difference in the intraoperative use of dexmedetomidine hydrochloride between the two groups.

\section{Treatment efficacy}

A comparison of treatment effects is summarized in Table 2. The sedation index is summarized in Table 3, and adverse events are summarized in Table 4. There was no significant difference in the rate of recurrent $\mathrm{AF}$ between the buprenorphine and fentanyl groups 1 year after surgery.

There was also no significant difference in postoperative complications, the exposure to the cumulative dose area product, and cumulative total air kerma between the two groups.

As an indicator of safety with different analgesics, intraoperative BIS values were $82.8(59.0-95.5)$ in the buprenorphine group and significantly lower, 76.9 (62.5-95.6), in the fentanyl group $(p=0.02)$, but the RASS score was not significantly different between the two groups.

The median number of additional doses of thiamylal sodium, an indicator of intraoperative pain and discomfort, was similar to that of the fentanyl group (once, [0$4]$ ) and the buprenorphine group (once [0-6]; $\mathrm{p}=<$ 0.01 ), but the distribution was less in the fentanyl group, and the difference was significant. There was no significant difference in adverse events (postoperative vomiting) between the two groups. There was also no significant difference in metoclopramide hydrochloride administration.

\section{Discussion}

In the present study, we examined whether different anesthesia management agents used for ablation affect outcomes in terms of efficacy (the rate of recurrence and complications of AF (atrioventricular block, cardiac

Table 1 Characteristics of the patients who underwent ablation

\begin{tabular}{|c|c|c|c|}
\hline & Buprenorphine $(n=46)$ & Fentanyl $(n=47)$ & $p$ value \\
\hline Age (years) mean $\pm S D$ & $64.5 \pm 10.2$ & $63.6 \pm 9.1$ & 0.66 \\
\hline $\operatorname{Sex}(M / F)$ & $35 / 11$ & $32 / 15$ & 0.49 \\
\hline Body mass index $\left(\mathrm{kg} / \mathrm{m}^{2}\right)$ mean $\pm \mathrm{SD}$ & $24.8 \pm 4.2$ & $23.9 \pm 5.4$ & 0.67 \\
\hline $\mathrm{CHADS}_{2}$ score median (min-max) & $1(0-4)$ & $1(0-5)$ & 0.99 \\
\hline $\mathrm{CHA}_{2} \mathrm{DS}_{2}$-VASc score median (min-max) & $2(0-5)$ & $2(0-7)$ & 0.87 \\
\hline Creatinine clearance $(\mathrm{mL} / \mathrm{min})$ mean $\pm \mathrm{SD}$ & $86.3 \pm 27.1$ & $81.2 \pm 26.7$ & 0.36 \\
\hline Left atrial dimension $(\mathrm{LAD})(\mathrm{cm})$ mean $\pm S D$ & $3.71 \pm 0.57$ & $3.86 \pm 0.65$ & 0.27 \\
\hline Left ventricular ejection fraction (LVEF) (\%) mean \pm SD & $69.24 \pm 6.15$ & $63.89 \pm 9.19$ & $<0.01$ \\
\hline Average Drug Use $(\mathrm{mg})$ mean \pm SD & $0.18 \pm 0.05$ & $1.06 \pm 0.35$ & - \\
\hline Dexmedetomidine hydrochloride $(\mu \mathrm{g})$ median (min-max) & $107.2(46.0-201.6)$ & $117.2(61.6-282.4)$ & 0.14 \\
\hline Patients who received cardioversion No. of patients (percentage of patients) & $25(54.3 \%)$ & $26(55.3 \%)$ & 1.00 \\
\hline \multicolumn{4}{|l|}{ Type of AF } \\
\hline Paroxysmal. No. of patients (percentage of patients) & $31(67.4 \%)$ & $29(61.7 \%)$ & \multirow[t]{3}{*}{0.83} \\
\hline Persistent No. of patients (percentage of patients) & $13(28.2 \%)$ & $15(31.9 \%)$ & \\
\hline Long lasting persistent No. of patients (percentage of patients) & $2(4.3 \%)$ & $3(6.4 \%)$ & \\
\hline \multicolumn{4}{|l|}{ Ablation method } \\
\hline PVI No. of patients (percentage of patients) & $31(67.4 \%)$ & $31(66.0 \%)$ & \multirow[t]{3}{*}{0.45} \\
\hline $\mathrm{PVI}+\mathrm{CTI}$ ablation No. of patients (percentage of patients) & $9(19.6 \%)$ & $13(27.7 \%)$ & \\
\hline Other No. of patients (percentage of patients) & $6(13.0 \%)$ & $3(6.4 \%)$ & \\
\hline
\end{tabular}

No. number, PVI Pulmonary vein isolation, CTI cavo-tricuspid isthmus line 
Table 2 Treatment outcomes and exposure doses

\begin{tabular}{llll}
\hline & $\begin{array}{l}\text { Buprenorphine } \\
(\boldsymbol{n}=\mathbf{4 6})\end{array}$ & $\begin{array}{l}\text { Fentanyl } \\
(\boldsymbol{n}=\mathbf{4 7})\end{array}$ & $\boldsymbol{p}$ value \\
\hline Recurrence of AF No. of patients (percentage of patients) & $10(21.7 \%)$ & $14(29.8 \%)$ & 0.48 \\
Complications No. of patients (percentage of patients) & $0(0 \%)$ & $1(2.1 \%)^{\mathrm{a}}$ & 1.00 \\
Cumulative DAP (fluoroscopy) (mGycm ${ }^{2}$ ) median (min-max) & $22,298.5(9749-188,652)$ & $25,100.0(8750-95,081)$ & $3260(166-11,013)$ \\
Cumulative DAP (exposure) (mGycm2) median (min-max) & $3779(109-22,622)$ & $268.5(84.9-1105.0)$ & 0.38 \\
Cumulative total air kerma (mGy) median (min-max) & $286.4(95.3-2192.4)$ & & 0.51 \\
\hline
\end{tabular}

a Pseudoaneurysm

$A F$ atrial fibrillation, $D A P$ dose area product

tamponade, fistula in left esophagus, and others)) and safety (depth of sedation, number of additional doses of thiamylal sodium administered, and frequency of vomiting). The number of additional doses of thiamylal sodium, an indicator of intraoperative pain and discomfort, administered were significantly lower in the fentanyl group than in the buprenorphine group. The average usage of buprenorphine hydrochloride in the buprenorphine group was $0.18 \mathrm{mg}$. In contrast, the average usage of fentanyl citrate in the fentanyl group was $1.06 \mathrm{mg}$. In the Pharmaceutical Guidelines of the Japanese Society of Anesthesiologists [9], the respective analgesic effects of buprenorphine hydrochloride and fentanyl citrate are approximately 33-40 times and 50-100 times that of morphine.

Fentanyl citrate generally has a titer twice of that of buprenorphine hydrochloride, indicating that fentanyl citrate is used in higher quantities. The BIS was significantly lower in the fentanyl group, but the difference was small (approximately 3.8 ) and could be considered of minor clinical significance. Both groups were in the category of deep to mild sedation. There was no difference in the RASS scores between the two groups, and the sedative effect was considered to be nearly equivalent. The use of fentanyl citrate as an analgesic in sufficient quantities is expected to reduce intraoperative discomfort, pain, and body movement without any deeper sedation than necessary, representing a more reliable and safe option for the physician. However, with respect to postoperative vomiting, there was no significant difference between the two groups of patients who received metoclopramide as an antiemetic agent. In a metaanalysis of opioid adverse events [10], vomiting was more common with buprenorphine hydrochloride than with fentanyl citrate.

Buprenorphine hydrochloride is a long-acting drug (with a half-life of approximately $10 \mathrm{~h}$ ). Conversely, fentanyl citrate is shorter acting (with a half-life of $30 \mathrm{~min}$ to $1 \mathrm{~h}$ ) [9]. Hence, we switched from buprenorphine hydrochloride to fentanyl citrate considering that the effects of fentanyl citrate would dissipate while the effects of dexmedetomidine hydrochloride would remain, thereby reducing postoperative vomiting. In a previous study using fentanyl as an analgesic [11], approximately $13 \%$ of patients were found to have vomiting, and the frequency of vomiting in the fentanyl group was as expected. There was no report of buprenorphine use during ablation, but the frequency of vomiting that occurred from other uses varied widely, ranging from a few percent to approximately $50 \%$, as reported [12-15]. In this study, there was a difference in vomiting (approximately $30 \%$ in the buprenorphine group and approximately $15 \%$ in the fentanyl group), but the difference was not statistically significant. One possible explanation for the lack of a significant difference between the two groups was the higher use of fentanyl compared to that of buprenorphine.

The most important outcomes of AF ablation to consider are the rate of AF recurrence and complications of AF. A previous study [16] reported a recurrence rate of $30-50 \%$ at 1 year postoperatively for AF. Although the current study was conducted with a small group, the recurrence rate was not significantly different from the previous study. There were few complications in this study, and they were well managed. Therefore, the combination of fentanyl citrate, thiamylal sodium, and

Table 3 Sedation index during ablation

\begin{tabular}{llll}
\hline & Buprenorphine $(\boldsymbol{n}=\mathbf{4 6})$ & Fentanyl $(\boldsymbol{n}=\mathbf{4 7})$ & $\boldsymbol{p}$ value \\
\hline Average BIS median (min-max) & $82.8(59.0-95.5)$ & $76.9(62.5-95.6)$ & 0.02 \\
Average RASS score median (min-max) & $-1.38(-4.00-0.00)$ & $-2.00(-4.00-0.00)$ & 0.11 \\
No. of additional administrations of thiamylal median (min-max) & $1(0-6)$ & $1(0-4)$ & $25(0-250)$ \\
Thiamylal Sodium usage (mg) median (min-max) & $75(0-450)$ & $<0.01$ \\
\hline
\end{tabular}

BIS bispectral index, No. number, RASS Richmond Agitation-Sedation Scale 
Table 4 Adverse events and antiemetic administration after ablation

\begin{tabular}{|c|c|c|c|}
\hline & $\begin{array}{l}\text { Buprenorphine } \\
(n=46)\end{array}$ & $\begin{array}{l}\text { Fentanyl } \\
(n=47)\end{array}$ & $p$ value \\
\hline No. of patients with vomiting No. of patients (percentage of patients) & $14(30.4 \%)$ & $7(14.9 \%)$ & 0.09 \\
\hline No. of patients receiving prophylactic metoclopramide hydrochloride No. of patients (percentage of patients) & $31(67.4 \%)$ & $24(51.1 \%)$ & 0.14 \\
\hline
\end{tabular}

No. number

dexmedetomidine hydrochloride may be a useful option in the management of anesthesia during AF ablation, particularly because of the reduction of intraoperative body movements with this approach.

This study has potential limitations. All patients in the present study underwent radiofrequency ablation; therefore, it is unclear whether our results can be extrapolated to the management of anesthesia during cryoablation or hot balloon ablation procedures. Additionally, the use of different catheter and respiratory management devices from one facility to the next may result in varied outcomes. As this study was carried out retrospectively at a single center with a small sample size, future randomized comparative trials on a larger scale would be necessary.

\section{Conclusions}

In conclusion, both fentanyl citrate and buprenorphine hydrochloride are useful in the management of anesthesia during AF ablation, suggesting that the use of fentanyl citrate may decrease intraoperative patient motion and discomfort.

\section{Abbreviations}

AF: Atrial fibrillation; BIS: Bispectral index; BMI: Body mass index; Ccr: Creatinine clearance; CTI: Cavo-tricuspid isthmus line; DAP: Dose area product; LAD: Left atrial dimension; LVEF: Left ventricular ejection fraction; PVI: Pulmonary vein isolation; RASS: Richmond Agitation-Sedation Scale

\section{Acknowledgments}

We would like to thank Editage (www.editage.com) for English language editing.

\section{Authors' contributions}

TK, DS, and MH conceived and designed this study. DS, NT, and MA assisted with the research design. TK provided epidemiological data and performed the statistical analyses. NT, TS, and MA assisted with performing the statistical analyses. TK, NT, and MH wrote the manuscript. All authors have read and approved the final version of the manuscript.

\section{Funding}

There are no funding sources for this study.

\section{Availability of data and materials}

The datasets analyzed during the current study are available from the corresponding author on reasonable request.

\section{Ethics approval and consent to participate}

This study was carried out following the Helsinki Declaration, the "Medical Guidelines for Medical and Health Research Involving Human Subjects," and the "Guide for the appropriate handling of personal information for medical and nursing care professionals." Approval was obtained from this hospital's Ethics Review Committee, and adequate consideration was given for the protection of personal data (Approval No. 517).
Consent for publication

Not applicable.

\section{Competing interests}

The authors declare that they have no competing interests.

\section{Author details}

'Department of Pharmacy, Kariya Toyota General Hospital, 5-15, Sumiyoshi-cho, Kariya City, Aichi Prefecture 448-8505, Japan. ${ }^{2}$ Department of Cardiology, Kariya Toyota General Hospital, 5-15, Sumiyoshi-cho, Kariya City, Aichi Prefecture 448-8505, Japan.

Received: 3 September 2020 Accepted: 26 November 2020

Published online: 04 January 2021

\section{References}

1. Friberg $L$, Tabrizi F, Englund A. Catheter ablation for atrial fibrillation is associated with lower incidence of stroke and death: data from Swedish health registries. Eur Heart J. 2016;37:2478-87.

2. Marrouche NF, Brachmann J, Andresen D, Jürgen Siebels J, Boersma L, Jordaens L, et al. CASTLE-AF investigators. Catheter ablation for atrial fibrillation with heart failure. N Engl J Med. 2018;378:417-27.

3. Packer DL, Mark DB, Robb RA, Monahan KH, Bahnson TD, Poole JE, et al. Effect of catheter ablation vs antiarrhythmic drug therapy on mortality, stroke, bleeding, and cardiac arrest among patients with atrial fibrillation: the CABANA randomized clinical trial. JAMA. 2019;321:1261-74.

4. Chikata A, Kato T, Yaegashi T, Sakagami S, Kato C, Saeki T, et al. General anesthesia improves contact force and reduces gap formation in pulmonary vein isolation: a comparison with conscious sedation. Heart Vessel. 2017;32: 997-1005.

5. Di Biase L, Conti S, Mohanty P, Bai R, Sanchez J, Walton D, et al. General anesthesia reduces the prevalence of pulmonary vein reconnection during repeat ablation when compared with conscious sedation: results from a randomized study. Heart Rhythm. 2011;8:368-72.

6. The Japanese Circulation Society. 2018 JCS/JHRS Guideline on NonPharmacotherapy of Cardiac Arrhythmias 2019. www.j-circ.or.jp/guideline/ pdf/JCS2018_kurita_nogami.pdf.

7. Schultz A, Siedenberg M, Grouven U, Kneif T, Schultz B. Comparison of Narcotrend index, Bispectral index, spectral and entropy parameters during induction of propofol-remifentanil anaesthesia. J Clin Monit Comput. 2008; 22:103-11.

8. Kanda Y. Investigation of the freely available easy-to-use software 'EZR' for medical statistics. Bone Marrow Transplant. 2013:48:452-8.

9. Japanese Society of Anesthesiologists. Usage Guidelines for Anesthesia and Anesthesia-related Drugs. Third Edition. 2019. https://anesth.or.jp/users/ person/guide_line/medicine Accessed 04 Sept 2020.

10. Dinges HC, Otto S, Stay DK, Bäumlein S, Waldmann S, Kranke P, et al. Side effect rates of opioids in equianalgesic doses via intravenous patientcontrolled analgesia: a systematic review and network meta-analysis. Anesth Analg. 2019;129:1153-62

11. Tyndall A, Nyström KV, Funk M. Nausea and vomiting in patients undergoing radiofrequency catheter ablation. Am J Crit Care. 1997;6:437-44.

12. Kay B. A double-blind comparison of morphine and buprenorphine in the prevention of pain after operation. Br J Anaesth. 1978;50:605-9.

13. Green DW, Sinclair JR, Mikhael MS. Buprenorphine versus morphine. A comparison of intra-operative and postoperative analgesia. Anaesthesia. 1985;40:371-5.

14. Van den Berg AA, Honjol NM, Prabhu NV, Datta S, Rozario CJ, Muraleedaran $R$, et al. Analgesics and ENT surgery. A clinical comparison of the intraoperative, recovery and postoperative effects of buprenorphine, diclofenac, fentanyl, morphine, nalbuphine, pethidine and placebo given 
intravenously with induction of anaesthesia. Br J Clin Pharmacol. 1994;38: 533-43.

15. Bradley JP. A comparison of morphine and buprenorphine for analgesia after abdominal surgery. Anaesth Intensive Care. 1984;12:303-1.

16. Andrade JG, Champagne J, Dubuc M, Deyell MW, Verma A, Marcle L, et al.

Cryoballoon or radiofrequency ablation for atrial fibrillation assessed by

continuous monitoring: a randomized clinical trial. Circulation. 2019;140: 1779-88.

\section{Publisher's Note}

Springer Nature remains neutral with regard to jurisdictional claims in published maps and institutional affiliations.

Ready to submit your research? Choose BMC and benefit from:

- fast, convenient online submission

- thorough peer review by experienced researchers in your field

- rapid publication on acceptance

- support for research data, including large and complex data types

- gold Open Access which fosters wider collaboration and increased citations

- maximum visibility for your research: over $100 \mathrm{M}$ website views per year

At $B M C$, research is always in progress.

Learn more biomedcentral.com/submissions 